\title{
Research of Comprehensive Effect Evaluation of Organic Agriculture Cluster Based on the Fuzzy Co-Clustering for Images
}

\author{
Lankai Liu $^{\mathrm{a}}$ and Qianqian Zhang ${ }^{\mathrm{b}}$ \\ School of Quality Development Institute, Kunming University of \\ Science and Technology, Kunming 650093, China \\ aZQQ_WX_529@163.com, b1151679297@qq.com
}

Keywords: organic agriculture cluster, fuzzy co-clustering for images, comprehensive effect, evaluation research.

\begin{abstract}
Organic agriculture cluster is the trend of the future development of organic agriculture in China. In order to scientific and reasonable evaluation of organic agriculture cluster comprehensive effect, according to the research status of agricultural cluster effect and the characteristics of organic agriculture cluster, the comprehensive effect of organic agriculture cluster is divided into three subsystems: economic effects, social effects and ecological effects. Drawing on the existing agricultural cluster effect evaluation system, it establishes evaluation system of a 3 levels and 26 specific indexes of organic agriculture cluster effect to provide certain theoretical basis for the development of organic agriculture cluster based on the fuzzy co-clustering for images.
\end{abstract}

\section{Introduction}

With the expansion of the organic agriculture market and the enhancement of competitiveness, enterprises related to organic agriculture have gradually begun to cluster development, which not only helps to promote the leapfrog development of related enterprises, but also enhances the competitiveness of organic agriculture cluster regional economy. The organic agriculture cluster is a new development mode of agricultural cluster in China at present, and the formation of the cluster effect is usually the result of the cluster economy comprehensive function[1]. From the view of system dynamics, the cluster effect is the important embodiment of the cluster competitive advantage, and it is also the basic guarantee for the sustainable development of the industrial cluster[2]. According to the unified consensus that many scholars have formed on the cluster effect, the author defines the organic agriculture cluster effect as follows: farmers, enterprises and institutions related to organic agriculture are clustered in specific geographical areas, which makes the farmers, enterprises and units in the cluster have the competitive advantages of low cost, differentiation and innovation. In addition to the number agglomeration of organic agro-industrial cluster, it is more important that each organic farmer, enterprise and organization in the cluster has a network-like formation and a special cooperative and competitive relationship, which can promote the optimal allocation of resources to the maximum extent within the cluster.

Because there are few literatures on the comprehensive effect of organic agriculture cluster, the author draws up the evaluation index system of the comprehensive effect of organic agriculture cluster by drawing lessons from the research results of agricultural cluster effect. The research on the effect of agricultural cluster is as follows. Many scholars have analyzed the effect of agricultural cluster from different angles and different aspects. For example, Zhu Q.H. et al. (2004) analyzed the cluster effect of agricultural science and technology parks from technological innovation effect, competitive advantage effect and spatial location effect[3]. Wang B.L. et al. (2006) put forward the concept of green agricultural cluster and analyzed the green fruit industry cluster effect of Weibei in Shanxi Province from the four aspects of technological innovation, competition, spatial location and ecological effects by using non-monetary evaluation and analytic hierarchy process (AHP)[4]. Fu X.SH. (2007), based on reviewing the competitive advantages of industrial cluster, proposed that the evaluation index of industrial cluster comprehensive effect should be decomposed into three sub-indexes, such as efficiency, market competitive position and structure[5]. Su Z.F. et al. (2009) 
analyzed the sucrose industry cluster effect in Guangxi from the aspects of industrial scale, scope economy, transaction cost, competitiveness, knowledge spillover and innovation effect[6]. Yan SH.F. (2010) used qualitative methods to analyze the relationship between animal husbandry industry agglomeration and farmers' income level, agricultural industry structure, agricultural competitiveness, and agricultural urbanization[7]. Wang Y.R. (2012) analyzed the agricultural cluster effect from industry development effect, technology development effect and social development effect by using the Cobb-Douglas production function and vector auto regression (VAR) method[8]. Xu X. (2013) selected four indexes of industry concentration (CRn) and location quotient coefficient (LQ), the gene coefficient (G) and spatial concentration index (EG) for analysis of Hunan agricultural cluster effect[9]. Zhou X.D. (2013) applied qualitative methods to analyze the effect of green agricultural cluster from economic, social and ecological effect[10]. Ge X.J. (2014) analyzed Shouguang vegetable industry cluster in promoting rural economic development level and scale economy effect by using theoretical analysis and empirical analysis method[11]. Li J.L. et al. (2016) proposed a method of TOPSIS evaluation for industrial cluster development effect based on interval number evaluation scale[12].

Therefore, according to the study of agricultural cluster effect and the characteristics of organic agriculture, the comprehensive effect of organic agriculture cluster is divided into economic effect, social effect and ecological effect. This article will establish and optimize the evaluation index system of the comprehensive effect of the organic agriculture cluster by using the Fuzzy CoClustering for Images to provide some theoretical basis for the development of organic agriculture cluster.

\section{Methods and Initial evaluation index system}

\subsection{Methods}

The Fuzzy Co-Clustering for Images (FCCI) is a dynamic clustering algorithm based on the fuzzy clustering methods (FCM). The difference with FCM is that FCCI not only takes into account the correlation of samples, but also considers the correlation of sample attributes. So, FCCI algorithm introduces two membership matrices, namely, the sample degree membership $u_{c i}$ and the attribute ranking membership $v_{c j}$, which makes the samples and attributes have fuzziness in the clustering process. That is to say, it makes the samples belong to different classes to be in line with the true distribution of data in the process of clustering. FCCI algorithm uses the weighted Euclidean distance between samples and the center of mass to reflect the aggregation degree of clustering results, so the minimum value of the objective function is obtained. The FCCI objective function $J_{F C C I}$ is as follows.

$$
J_{F C C I}=\sum_{c=1}^{C} \sum_{i=1}^{N} \sum_{j=1}^{K} u_{c i} v_{c j} D_{c i j}+T_{u} \sum_{c=1}^{C} \sum_{i=1}^{N} u_{c i} \log u_{c i}+T_{v} \sum_{c=1}^{C} \sum_{j=1}^{K} v_{c j} \log v_{c j}
$$

The minimum value of the objective function should be satisfied the following two constraints.

$$
\begin{aligned}
& \sum_{c=1}^{C} u_{c i}=1, u_{c i} \in[0,1], \forall i=1, \ldots N \\
& \sum_{j=1}^{K} v_{c j}=1, v_{c j} \in[0,1], \forall c=1, \ldots C
\end{aligned}
$$

The first term in equation (1) represents the effective squared distance sum. When the first item reaches the minimum value, the distance to the center of mass is given a higher degree of membership, and the attribute with stronger correlation is given a higher weight. $D_{c i j}$ is the Euclidean distance between the sample $x_{i j}$ and the centroid $p_{c j}$, namely, $D_{c i j}=\left(x_{i j}-p_{c j}\right)^{2}$. The second and third items are the regularized information entropy of $u_{c i}$ and $v_{c j}$, which is helpful to the fuzziness of clustering results. $T_{u}$ and $T_{v}$ are user-defined weighting parameters, which are used to control the fuzzy degree of clustering, and the higher the value, the greater the degree of fuzzy. 


\subsection{Data source}

The samples of the study are collected from 7 counties (districts) in Yunnan Province, which are awarded the "Organic Certification Brand Demonstration Zone", including Simao, Menghai, Binchuan, Tengchong, Yongsheng, Cangyuan and Yangbi counties. All indexes' data are derived from these seven counties (districts) in 2015 the statistical yearbook, the 2010-2015 annual report of the county government work and the Statistical Yearbook of Yunnan Province (2015), and part of the indexes is calculated by using the original data.

\subsection{Initial evaluation index system}

In China, the development of organic agricultural cluster is relatively late, so far there is no systematic and complete evaluation index system of the comprehensive effect of organic agriculture cluster. However, the rise of agricultural cluster is relatively early and many scholars have formed a certain theoretical framework for the study of its effect. Therefore, according to the scholars' research on the evaluation system of agricultural cluster effect and following the principles of systematic, comprehensive, scientific, comparability, operability, representativeness and accessibility of the evaluation index system, this article will establish the evaluation index system of the comprehensive effect of organic agriculture cluster, including 3 first-level indexes and 26 secondary initial indexes. These specific indexes are as follows:

Economic effect C1: it mainly includes the economic contribution rate of organic industry E1, economic efficiency of organic industry E2, production cost advantage E3, market recognition advantage $E 4$, product difference advantage $E 5$, scale economies advantage $E 6$, scope economies advantage $E 7$, regional brand advantages $E$ 8, urbanization advantages $E 9$, intermediary services E10 and policy advantage E11.

Social effect C2: it mainly includes the advantage of knowledge spillover $E 12$, the availability of innovative resources $E 13$, the catching and drawing advantage $E 14$, the personnel growth rate of organic agriculture $E 15$, the quality of organic agriculture personnel level $E 16$, the increase degree of organic farmer income E17.

Ecological effect C3: it mainly includes the investment of environmental protection $E 18$, the farmers' awareness of environmental protection $E 19$, the compliance rate of organic agricultural products $E 20$, the material recycling rate $E 21$, the pest incidence $E 22$, the soil erosion area ratio $E 23$, the forest vegetation coverage E24, pollution load index E25, soil quality E26.

Considering that there are too many evaluation indexes in the building evaluation index system process of the comprehensive effect of organic agriculture cluster, there may be a great correlation between the indexes. So, this article combines FCCI method to gather the strong correlative indexes together into a comprehensive index class, and use the comprehensive index to evaluate the comprehensive effect of organic agriculture cluster.

\section{4 classification number}

The number of cluster centroid about FCCI and other traditional clustering algorithm is determined by experience or a large number of experiments to get the optimal value. And it has a certain degree of blindness. Because of the above reasons, the author puts forward a method based on principal component analysis to determine the number of clusters, and then to analyze the samples data by FCCI to achieve the purpose of clustering. The principal component analysis method is used to determine the number of FCCI clusters in economic effect, social effect and ecological effect, respectively.

Step 1: Original data preprocessing and testing

The collected data reflects the different information about the organic agriculture cluster in the 7 organic certification brand demonstration area. In order to eliminate the interference caused by the different dimensions and different orders of magnitude, the original data must be dimensionless processing. At the beginning of principal component analysis, the author selects the standardized method in SPSS 22.0 to deal with the original data. The results by KMO test and Bartlett test with the standardized data are following: the value of KMO is 0.625 ( more than 0.5 ) and the significant level of Bartlett is less than 0.05. Namely the evaluation indexes are independent of each other, which is proved to be suitable for the principal component analysis. 
Step 2: Extract common factors

For the determining the number of extracted principal components, the author uses the method of combining eigenvalue criteria and factor cumulative variance contribution. That is, selecting principal components with eigenvalues greater than or equal to 1 as common factors, and the number of factors satisfying variance cumulative contribution rate $85 \%$ or more. The results are shown in Table 1 through SPSS 22.0.

Table 1 Eigenvalues of the main factors, the proportion of the contribution rate and cumulative contribution rate

\begin{tabular}{cccccccc}
\hline & & \multicolumn{3}{c}{ Initial eigenvalue } & \multicolumn{2}{c}{ Extraction square and loading } \\
\cline { 3 - 7 } $\begin{array}{c}\text { First-level } \\
\text { index }\end{array}$ & Ingredients & Eigenvalues & $\begin{array}{c}\text { Variance } \\
\text { contribution } \\
\text { rate (\%) }\end{array}$ & $\begin{array}{c}\text { Cumulative } \\
\text { variance } \\
\text { contribution } \\
\text { rate (\%) }\end{array}$ & $\begin{array}{c}\text { Variance } \\
\text { Eigenvalues }\end{array}$ & $\begin{array}{c}\text { Cumulative } \\
\text { variance } \\
\text { contribution } \\
\text { rate (\%) }\end{array}$ & $\begin{array}{c}\text { contribution } \\
\text { rate(\%) }\end{array}$ \\
\hline Economic & 1 & 7.064 & 45.531 & 45.531 & 7.064 & 45.531 & 45.531 \\
effect C1 & 3 & 5.389 & 25.643 & 71.174 & 5.389 & 25.643 & 71.174 \\
& 4 & 2.163 & 10.084 & 82.014 & 2.163 & 10.084 & 82.014 \\
\hline Social & 5 & 1.155 & 10.007 & 91.265 & 1.155 & 10.007 & 91.265 \\
\hline effect C2 & 6 & 1.179 & 54.874 & 54.874 & 3.179 & 54.874 & 54.874 \\
Ecological & 7 & 4.526 & 50.289 & 50.289 & 4.526 & 50.280 & 85.074 \\
effect C3 & 8 & 2.890 & 26.037 & 76.326 & 2.890 & 26.037 & 76.326 \\
\hline
\end{tabular}

Table 1 is shown that the economic, social and ecological effects can be described by four common factors, two common factors and three common factors. Their eigenvalues are greater than 1 , and the cumulative contribution rates are $91.265 \%, 85.074 \%, 87.566 \%$. All are more than $85 \%$, which indicates that the primitive variables on economic effect, the social effect and ecological effect have better explanation ability. So when the fuzzy variables are clustered, the economic effect, social effect and ecological effect are clustered into four classes, two classes and three classes, respectively.

\section{Optimization index system}

\subsection{Data standardization process}

The normalization of the original data is due to the differences between the original dimension data and the magnitude units. In order to make the comparison of data from different dimensions and different orders of magnitude, it is usually necessary to transform the original data processing. Before using the FCCI analysis, the original data is processed by the method of central transformation in the standardized processing method. The central transformation is a transform that calculates the variance-covariance, which calculates the sample mean of each index variable at first and then subtracts the mean of the index variables from the original data. The equation is $x_{i j}^{*}=x_{i j}-\bar{x}_{j}(i=1,2, \ldots, n ; j=1,2, \ldots, m), \bar{x}_{j}=\sum x_{i j} / \mathrm{n}$. After standardization, the sum of each column data is 0 , and the sum of the squares of each column data is equal to the variance of the column data. At the same time, any difference in the covariance of the two column data is equal to the two column cross product's $1 /(n-1)$.

\subsection{Process analysis}

In the process of FCCI analysis, according to the minimum value of the objective function $J_{F C C I}$, the membership matrix is obtained. The indexes with strong correlation are gathered to achieve the goal of optimizing the comprehensive effect of the organic agriculture cluster. According to the results of principal component analysis, the initial cluster number $C$ of economic, social and ecological effects in the evaluation index of the comprehensive effect of organic agriculture cluster 
are set as 4, 2 and 3, respectively. In order to get the value of FCCI objective function $J_{F C C I}$, the main operation steps of using computer Java programming are as follows.

Step 1: Initialize parameters $T_{u}=10^{6}, T_{v}=10^{8}$, the maximum error $\varepsilon=10^{-5}$ and the maximum number of iterations $\tau_{\max }=100$, respectively.

Step 2: Set iteration number $\tau=1$.

Step 3: Initialize the sample degree membership $u_{c i}$ and the attribute ranking membership $v_{c j}$, which satisfy the condition of equation (2) and equation (3).

Step 4: REPEAT.

Step 5: Calculate the centroid $P_{c j}$ using equation (4).

$$
p_{c j}=\frac{\sum_{i=1}^{N} u_{c i} x_{i j}}{\sum_{i=1}^{N} u_{c i}}
$$

Step 6: Calculate the Euclidean distance $D_{c i j}$ using equation (5).

$$
D_{c i j}=d^{2}\left(x_{i j}, p_{c j}\right)=\left(x_{i j}-p_{c j}\right)^{2}
$$

Step 7: Calculate the attribute ranking membership $v_{c j}$ using equation (6).

$$
v_{c j}=\frac{\exp \left(-\sum_{i=1}^{N} u_{c i} D_{c i j} / T_{v}\right)}{\sum_{f=1}^{K} \exp \left(-\sum_{i=1}^{N} u_{c i} D_{c i f} / T_{v}\right)}
$$

Step 8: Calculate the sample degree membership $u_{c i}$ using equation (7).

$$
u_{c i}=\frac{\exp \left(-\sum_{j=1}^{K} v_{c j} D_{c i j} / T_{u}\right)}{\sum_{c=1}^{C} \exp \left(-\sum_{j=1}^{K} v_{c j} D_{c i j} / T_{u}\right)}
$$

Step 9:Calculate $\tau=\tau+1$. Continue step 4 until to satisfy the condition of $\tau=\tau_{\max }$ or $\max \left(\left|u_{c i}(\tau)-u_{c i}(\tau-1)\right|\right) \leq \varepsilon$ and then jump to the step 10 .

Step 10: Iterate over.

At last, according to the sample degree membership $u_{c i}$ of the comprehensive effect index variables of organic agriculture cluster, this paper uses the inverse fuzzification process to determine the specific classification of each index and to obtain the clustering result.

After several iterations, it can obtain the membership degree matrix $u_{c i}$ of the comprehensive effect of organic agriculture cluster. The result is in table 2 and the sum of membership degree of each index variable corresponding to different classes is 1 .

\subsection{Classification}

The membership degree matrix $u_{c i}$ obtained by FCCI analysis is used to judge the membership value of each index variable. The larger the value is, the closer the index variable is to this class. In order to enable us to clearly distinguish the class of the comprehensive effect index variable of organic agriculture cluster, according to the membership degree of each index variable in Table 2, the index classification results of economic effect, social effect and ecological effect are shown in Fig.1, Fig.2 and Fig.3.

As can be seen from Fig. 1 about economic effect, E1 and E2 belong to the first class CE1 named as the industrial development effect. E3, $E 5$ and $E 8$ belong to the second class $C E 2$ named as the competitive advantage effect. E4, E6, E7 and E9 belong to the third class CE3 named as the spatial location effect. E10 and E11 belong to the fourth class CE4 named as the urban scale effect. Therefore, the economic effect can be evaluated by industrial development effect, competitive advantage effect, spatial location effect and urban scale effect.

As can be seen from Fig.2 about social effect, E12, E13 and E14 are attributed to the fifth class CE5 named as the technological innovation effect. E15, E16, E17 and E18 are attributed to the sixth class CE6 named as the farmers' welfare effect. So, the social effect can be reflected by technological innovation effect and farmers' welfare effect.

As can be seen from Fig.3 about ecological effect, E19, E20 and E25 are affiliated to the seventh class CE7 named as the environmental improvement effect. E21 and E23 are affiliated to the eighth 
class CE8 named as the organic quality effect. E22, E24, E26 and E27 are affiliated to the ninth class CE9 named as the production condition effect. In a short, the ecological effect can be performed to environmental improvement effect, organic quality effect and production condition effect.

Table 2 the membership matrix $u_{c i}$

\begin{tabular}{|c|c|c|c|c|c|}
\hline \multirow{2}{*}{$\begin{array}{l}\text { First-level } \\
\text { index }\end{array}$} & \multirow{2}{*}{ Code } & \multicolumn{4}{|c|}{ The membership degree } \\
\hline & & The first class & The second class & The third class & The fourth class \\
\hline \multirow{11}{*}{$\begin{array}{l}\text { Economic } \\
\text { effect } C 1\end{array}$} & E1 & 0.36297 & 0.25431 & 0.20384 & 0.17888 \\
\hline & E2 & 0.33048 & 0.24716 & 0.22560 & 0.19676 \\
\hline & E3 & 0.16546 & 0.32859 & 0.27304 & 0.23291 \\
\hline & E4 & 0.26703 & 0.18644 & 0.40568 & 0.14085 \\
\hline & E5 & 0.15280 & 0.34766 & 0.21319 & 0.28635 \\
\hline & E6 & 0.25764 & 0.28053 & 0.30642 & 0.15541 \\
\hline & E7 & 0.13258 & 0.22459 & 0.35316 & 0.28976 \\
\hline & E8 & 0.18973 & 0.37156 & 0.25328 & 0.18543 \\
\hline & E9 & 0.24861 & 0.20172 & 0.29457 & 0.25510 \\
\hline & E10 & 0.26347 & 0.19342 & 0.24786 & 0.29525 \\
\hline & E11 & 0.18254 & 0.23356 & 0.25927 & 0.32463 \\
\hline \multirow{7}{*}{$\begin{array}{l}\text { Social effect } \\
\text { C2 }\end{array}$} & & The fifth class & The sixth class & & \\
\hline & E12 & 0.53347 & 0.46653 & - & - \\
\hline & E13 & 0.62594 & 0.37406 & - & - \\
\hline & E14 & 0.55161 & 0.44839 & - & - \\
\hline & E15 & 0.30924 & 0.69076 & - & - \\
\hline & E16 & 0.25482 & 0.74518 & - & 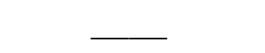 \\
\hline & E17 & 0.29985 & 0.70015 & - & - \\
\hline \multirow{10}{*}{$\begin{array}{l}\text { Ecological } \\
\text { effect } C 3\end{array}$} & & The seventh class & The eighth class & The ninth class & \\
\hline & E18 & 0.40783 & 0.36528 & 0.22689 & - \\
\hline & E19 & 0.38429 & 0.33529 & 0.28041 & - \\
\hline & E20 & 0.20316 & 0.47509 & 0.32175 & - \\
\hline & E21 & 0.19842 & 0.35964 & 0.44194 & - \\
\hline & E22 & 0.23758 & 0.50766 & 0.25476 & - \\
\hline & E23 & 0.38925 & 0.18640 & 0.42435 & - \\
\hline & E24 & 0.39637 & 0.31875 & 0.28488 & - \\
\hline & E25 & 0.29544 & 0.26629 & 0.43827 & - \\
\hline & E26 & 0.20376 & 0.33981 & 0.45643 & $\overline{-}$ \\
\hline
\end{tabular}

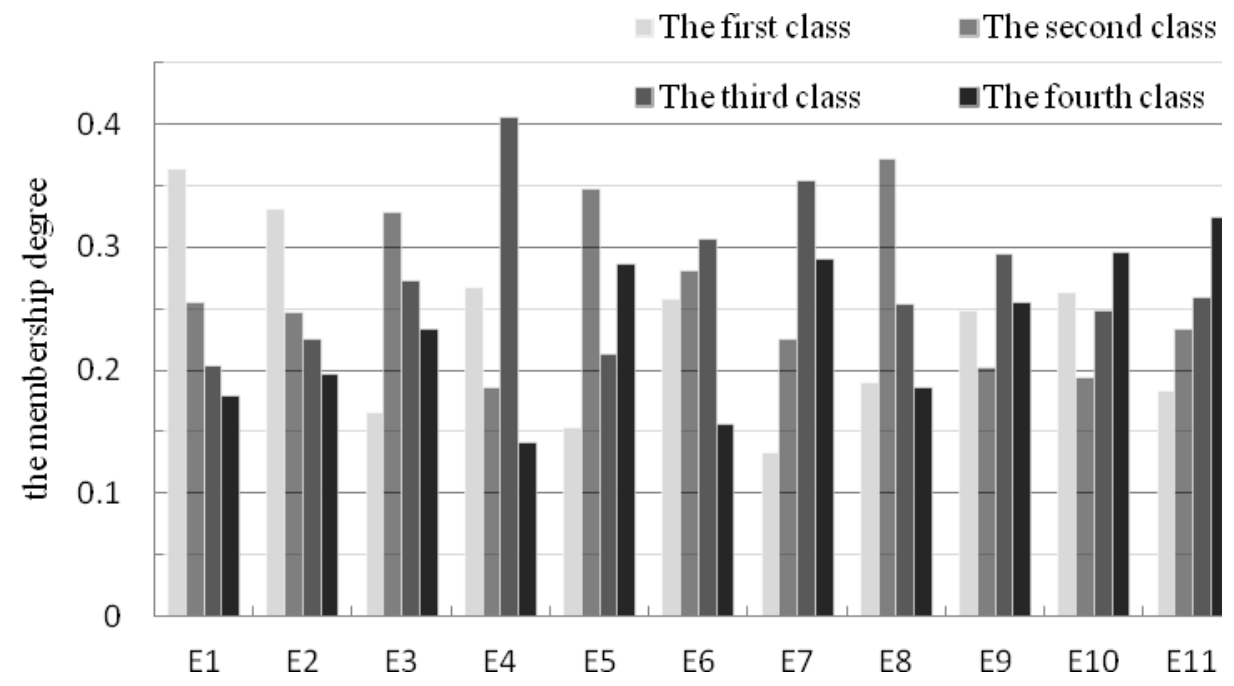

Fig.1 Economic effect index membership degree 


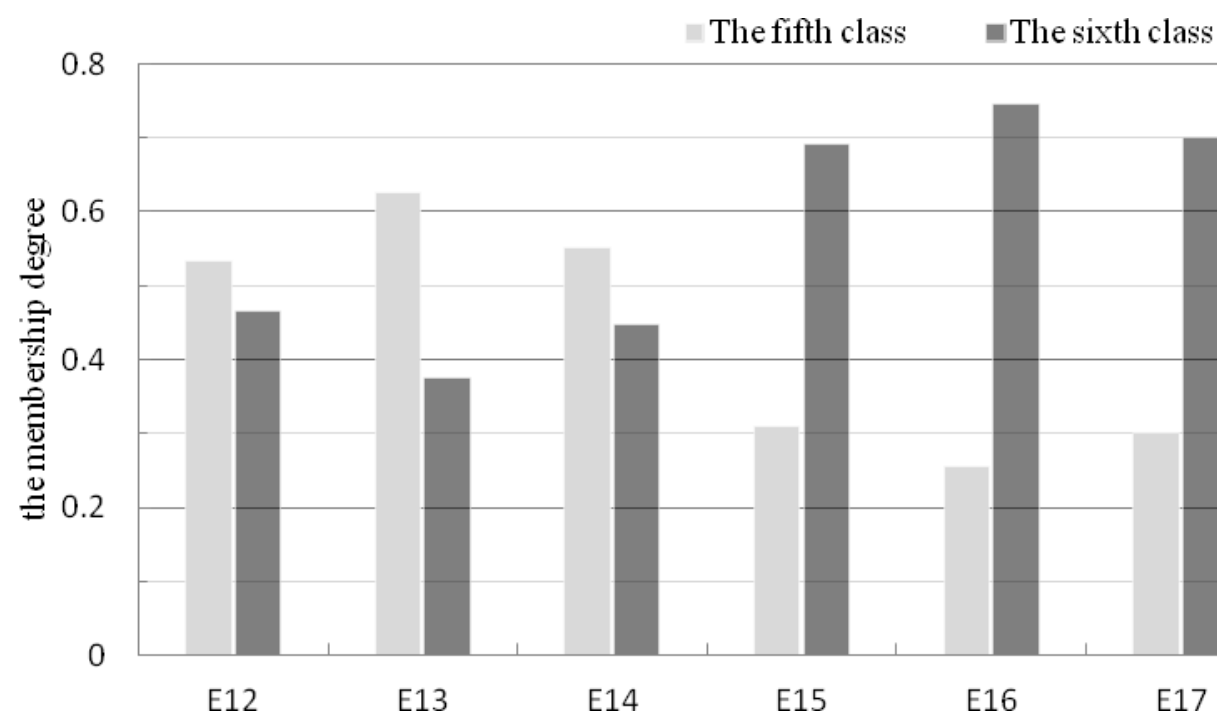

Fig.2 Social effect index membership degree

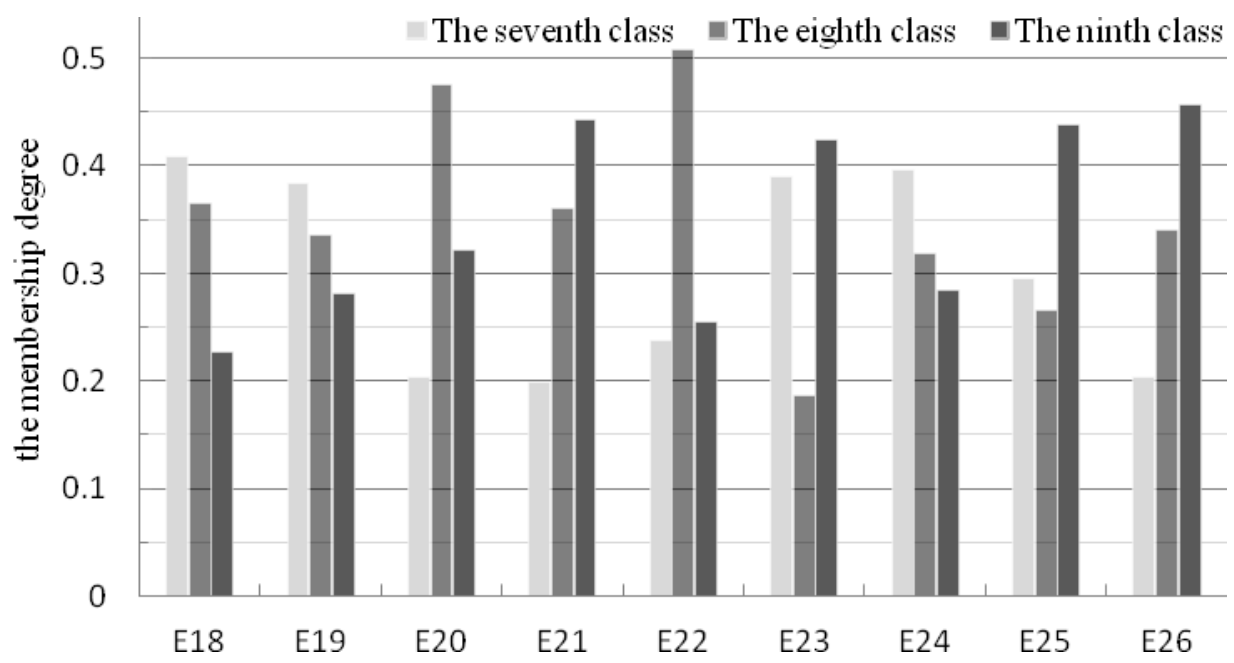

Fig.3 Ecological effect index membership degree

\subsection{Determine the index weight}

The membership degree matrix $u_{c i}$ of the FCCI analysis is normalized simply and can calculate the weight ratio of the comprehensive effect of organic agriculture cluster. Then it will use the simple linear weighting function to calculate the weight of the first-level index and the second-level index by equation (8) and the equation (9).

The first-level index weight:

$$
\left\{\begin{array}{l}
w_{C 1}=w_{C E 1}+w_{C E 2}+w_{C E 3}+w_{C E 4} \\
w_{C 2}=w_{C E 5}+w_{C E 6} \\
w_{C 3}=w_{C E 7}+w_{C E 8}+w_{C E 9}
\end{array}\right.
$$

The second-level index weight: 


$$
\left\{\begin{array}{l}
w_{C E 1}=\frac{u_{11}+u_{12}}{U} \\
w_{C E 2}=\frac{u_{23}+u_{25}+u_{28}}{U} \\
w_{C E 3}=\frac{u_{34}+u_{36}+u_{37}+u_{39}}{U} \\
w_{C E 4}=\frac{u_{410}+u_{411}}{U} \\
w_{C E 5}=\frac{u_{512}+u_{513}+u_{514}}{U} \\
w_{C E 6}=\frac{u_{615}+u_{616}+u_{617}}{U} \\
w_{C E 7}=\frac{u_{718}+u_{719}+u_{724}}{U} \\
w_{C E 8}=\frac{u_{820}+u_{822}}{U} \\
w_{C E 9}=\frac{u_{921}+u_{923}+u_{925}+u_{926}}{U}
\end{array}\right.
$$

The expression of $U$ :

$$
\begin{aligned}
& U=u_{11}+u_{12}+u_{23}+u_{25}+u_{28}+u_{34}+u_{36}+u_{37}+u_{39}+u_{410}+u_{411}+u_{512}+u_{513}+ \\
& u_{514}+u_{615}+u_{616}+u_{617}+u_{718}+u_{719}+u_{724}+u_{820}+u_{822}+u_{921}+u_{923}+u_{925}+u_{926}
\end{aligned}
$$

From the above we can see that the comprehensive effect index weight of organic agriculture cluster can be calculated and it is shown in Table 3 .

Table 3 The weight of the comprehensive effect evaluation index

\begin{tabular}{ccccccccccc}
\hline $\begin{array}{c}\text { The first-level } \\
\text { index }\end{array}$ & \multicolumn{2}{c}{$C 1$} & \multicolumn{2}{c}{$C 2$} & & \multicolumn{2}{c}{$C 3$} & \\
\hline weight & & \multicolumn{2}{c}{0.3236} & & 0.3345 & & 0.3419 & \\
\hline $\begin{array}{c}\text { The second-level } \\
\text { index }\end{array}$ & $C E 1$ & $C E 2$ & $C E 3$ & $C E 4$ & $C E 5$ & $C E 6$ & CE7 & CE8 & CE9 \\
\hline weight & 0.0603 & 0.0911 & 0.1182 & 0.0539 & 0.1488 & 0.1857 & 0.1033 & 0.0855 & 0.1531 \\
\hline
\end{tabular}

\section{Conclusions}

The organic agriculture is the latest industry and ecological effect of agricultural area. Whether it has formed a beginning industrial cluster and played the advantages of organic agriculture cluster is the key factor to determine the regional economy to achieve rapid development. From the whole point of view, the organic agriculture cluster in China is in the initial stage. There aren't yet the systematical methods of qualitative and quantitative research on the comprehensive effect of organic agriculture cluster. It is the urgent problem to be solved how to apply the effect theory of organic agricultural cluster to the development of agricultural regional policy and to be better to promote the rational distribution of the organic agriculture industry.

Based on the FCCI analysis method, this paper evaluates the comprehensive effect of the organic agriculture cluster and determines three first-level indexes and nine second-level indexes. As we can be seen from table 3, the proportion of the economic, social and ecological effects of organic agriculture cluster is $32.36 \%, 33.45 \%$, 34.19\%. Although the difference is very small, but it still reflects that the ecological effect of organic agriculture cluster occupies the most important position. The second is social effect and the third is economic effect. To a certain extent, it also shows that in today's rapid economic development, people not only focus on the pursuit of economic effect, but 
also pay more attention to the social effect and ecological effect agricultural development. The research on organic agriculture cluster effect has certain limitations. The results can not reflect the development of China's entire organic agriculture cluster because of the data selected only from Yunnan Province. We hope to provide a reference for the research of organic agriculture cluster effect in other areas of china.

\section{Acknowledgments}

This work was financially supported by the science and technology project of Yunnan province. The project title is the Study on Brand Building Model of Yunnan Plateau Characteristic Agricultural Cluster with Organic Certification, 2014RD025.

\section{References}

[1] G. B. Xu, K. Dong, T. Wang. A Study of Creative Industries Agglomeration, Technological Innovation Capacity and Regional Economic Growth-An Empirical Analysis of Anhui Province, J. East China Economic Management. 32(1) (2016) 83-86.

[2] X. H. Sun. Modeling and Simulation of Agglomeration Effect Using System Dynamics, J. Science of Science and Management of S.\& T. 29(4) (2008) 71-76.

[3] Q. H. Zhu, CH. G. Li. Analysis of Cluster Effect Based on Agricultural Science and Technology, J. Science and Technology Management Research. 24(2) (2004) 179-181.

[4] B. L. Wang, Y. T. Yao. Research and Application of Index System about Green Agriculture Industrial Cluster-Take Green Fruits Industrial Cluster of the North of Wei River in Shanxi Province, J. Chinese Agricultural Science Bulletin. 22(8) (2006) 588-593.

[5] X. SH. Fu. Research on evaluation index system of industrial cluster effect, J. The Science Education Article Collects. 11 (2008) 141-142.

[6] Z. F. Su, H. Yang, H. ZH. Liang. Analysis on the effect of sucrose industry cluster in Guangxi, J. Productivity Research. 24 (2009) 185-187.

[7] SH. F. Yan. Study on the effect of traditional animal husbandry industrial agglomeration, D. Northwest Agriculture and Forestry University. (2010).

[8] Y. R. Wang. Study on the effect and Countermeasure of agricultural industrial agglomeration, D. Hefei Polytechnic University. (2012).

[9] X. Xu, CH. Y. Liu. An Empirical Study on Assemble Effect of Agricultural Industrial Cluster in Hunan Province, J. Management of Agricultural Science and Technology. 32(4) (2013) 82-85.

[10] X. D. Zhou. The connotation, characteristics and effects of green agricultural industry cluster, J. Social Sciences in Hunan. 6 (2013) 141-144.

[11] X. J. Ge. An empirical study on rural economic development from the perspective of agglomeration effect, J. Rural Economy and Science-Technology. 25(4) (2014) 27-29.

[12] J. L. Li, T. Cheng. Evaluation Method of Food Industry Cluster Development Based on Interval Gray Incidence, J. Statistics \& Decision. 4(2016) 84-87. 